\title{
X-ray Imaging with Amorphous Silicon Active Matrix Flat-Panel Imagers (AMFPIs)
}

\author{
Youcef El-Mohri, Larry E. Antonuk, Kyung-Wook Jee, Manat Maolinbay, \\ Xiujiang Rong, Jeffrey H. Siewerdsen, Manav Verma and Qihua Zhao \\ Department of Radiation Oncology, University of Michigan Medical Center, Ann Arbor, Michigan 48109
}

\begin{abstract}
Recent advances in thin-film electronics technology have opened the way for the use of flat-panel imagers in a number of medical imaging applications. These novel imagers offer real time digital readout capabilities $(\sim 30$ frames per second), radiation hardness $\left(>10^{6} \mathrm{cGy}\right)$, large area $\left(30 \times 40 \mathrm{~cm}^{2}\right)$ and compactness $(\sim 1 \mathrm{~cm})$. Such qualities make them strong candidates for the replacement of conventional $\mathrm{x}$-ray imaging technologies such as film-screen and image intensifier systems. In this report, qualities and potential of amorphous silicon based active matrix flat-panel imagers are outlined for various applications such as radiation therapy, radiography, fluoroscopy and mammography.
\end{abstract}

The imagers under development by our group generally consist of a two-dimensional array of imaging pixels coupled to a dedicated external electronic acquisition system (1). The array is a glass substrate onto which amorphous silicon circuits have been deposited. Each pixel consists of a photodiode sensor coupled to a field-effect-transistor (FET). Incident radiation is detected indirectly by means of an overlying scintillator (e.g. phosphor or CsI). X-rays interacting in the scintillator generate light photons which are detected in the photodiodes. (An alternative AMFPI approach involves direct detection of the incident $\mathrm{X}$-rays by means of a thick converting material [e.g. a-Se] deposited over the FET matrix (2).) While the photodiodes act as storage capacitors for charge generated by the light photons, the FETs act as switches to enable the readout of this charge. During the course of our research dating back to 1987 , a series of progressively larger arrays has been developed starting from small $64 \times 40$ pixel devices (3). This work has advanced to the point where it has become possible to construct large area devices up to $30 \times 40 \mathrm{~cm}^{2}$. Array designs with pixel pitches ranging from $100 \mu \mathrm{m}$ to $508 \mu \mathrm{m}$ have been developed for various medical imaging applications such as radiation therapy, radiography and fluoroscopy.

In radiation therapy, successful treatment of cancer using an external beam of megavoltage ( $>1 \mathrm{MeV})$ photons is contingent on accurate patient positioning prior to and during the treatment, so that maximum dose is delivered to tumor cells while minimizing the dose to surrounding healthy tissue. To achieve this goal, we have developed imagers $(4,5)$ for this application which offer numerous advantages. Most recently, an $26 \times 26 \mathrm{~cm}^{2}$ imager, with a pixel pitch of $508 \mu \mathrm{m}$, has been developed $(5,6)$. Such AMFPI devices are well suited to the cancer therapy environment where high radiation doses are expected $\left(\sim 1 \times 10^{6} \mathrm{cGy}\right.$ per year). Previous studies demonstrated that amorphous silicon devices are highly radiation resistant and suffer only slight, or no degradation with exposure to high levels of radiation $(7,8)$. Also, the real-time readout offered by these imagers is ideal for rapid verification of patient positioning as compared to commonly used techniques involving film that require several minutes for film development. Recent observer-based studies have shown that performance achieved with these imagers is superior to that of conventional radiotherapy film systems (5).

In diagnostic imaging, where lower energy $\mathrm{x}$-rays are used, the demands for higher spatial resolution and the associated smaller signal sizes present more of a challenge. For radiographic imaging, which is the production and viewing of single $x$-ray images, and for fluoroscopic imaging, which is the real time production and viewing of continuous sequences of images, we have developed a prototype imager. This imager has a format of $1920 \times 1536$ pixels with a pixel pitch of 127 $\mu \mathrm{m}$ giving a total area of $19.5 \times 24.4 \mathrm{~cm}^{2}$ (9). Detailed characterization and performance assessment of this imager was performed for radiographic and fluoroscopic operation $(10,11)$ along with initial demonstrations of imaging with human subjects $(10,12)$. Although the performance of this imager is far from optimal, it is providing valuable information which, along with detailed theoretical analysis of the system (11), will allow the development of more optimized imagers in the future. For example, higher performance devices are possible through further improvements in thin-film processing

\section{CP417, Synchrotron Radiation Instrumentation: Tenth US National Conference} edited by Emest Fontes

(C) 1997 The American Institute of Physics 1-56396-742-1/97/\$10.00 
techniques such as the reduction of minimum feature sizes. Such improvements will make possible the creation of imagers suitable for the high resolution requirements of applications such as mammographic imaging. Overall, the potential advantages of AMFPIs for medical imaging (e.g. real-time digital readout, compactness, radiation resistance) are sufficiently numerous that there is very strong motivation to develop this technology to the point of matching or exceeding the imaging performance of established, highly optimized imaging technologies such as film-screen and image intensifiers.

In conclusion, our research suggests that active matrix, flat-panel imagers may well become a base technology in the 21 st century, providing superior radiation therapy imaging devices and potentially offering an alternative to current radiographic, fluoroscopic and mammographic imaging technologies.

\section{ACKNOWLEDGMENTS}

This work is supported by National Institutes of Health grants no. R01-CA51397 and R01-CA56135

\section{REFERENCES}

1. Morton, E.J., Antonuk, L.E.., Berry, J.E., Huang, W., Mody, P., and Yorkston, J., IEEE Trans. Nucl. Sci. 41, 1150-1154, (1994).

2. Zhao, W., and Rowlands, J.A., "A large area solid state detector for radiology using amorphous selenium", Proc. SPIE 1651, 134-143 (1992).

3. Street, R.A., Antonuk, L.E., and Perez-Mendez, V., "Amorphous silicon sensor arrays for radiation imaging", Mat. Res. Soc. Proc. 192, 441-452 (1990).

4. Antonuk, L.E., Yorkston, J., Huang, W., Sandler, H., Siewerdsen, J.H., and El-Mohri, Y., Int. J. Radiat. Onc. Biol. Phys. 36, 661-672 (1996).

5. Antonuk, L.E., El-Mohri, Y., Yorkston, J., Huang, W., Jee, K.W., Siewerdsen, J.H., Maolinbay, M., Scarpine, V.E., and Sandler, H., "Initial performance evaluation of an indirect-detection, active matrix flat-panel imager (AMFPI) prototype for megavoltage imaging", submitted to International Journal of Radiation Oncology, Biology, Physics.

6. Antonuk, L.E, Boudry, J., El-Mohri, Y., Huang, W., Siewerdsen, J.H., Yorkston, J., "Large area, flat-panel, amorphous silicon imagers", Proc. SPIE 2432, 216-227 (1995).

7. Boudry, J.M., and Antonuk, L.E, IEEE Trans. Nucl. Sci. 41, 703-707 (1994).

8. Boudry, J.M., and Antonuk, L.E., Med. Phys. 23, 743-754 (1996).

9. Antonuk, L.E., El-Mohri, Y., Huang, W., Jee, K.W., Maolinbay, M., Scarpine, V.E., Siewerdsen, J.H., Verma, M., Yorkston, J., and Street, R.A., "Development of a high resolution, active-matrix, flat-panel imager with enhanced fill factor", Proc. SPIE 3032, 2-13 (1997).

10. Antonuk, L.E., El-Mohri, Y., Siewerdsen, J.H., Yorkston, J., Huang, W., Scarpine, V.E., and Street, R.A., Med. Phys. 24, 51-70 (1997).

11. Siewerdsen, J.H., Antonuk, L.E., El-Mohri, Y., Yorkston, J., Boudry, J.M., and Huang, W., Med. Phys. 24, 71-89 (1997).

12. Antonuk, L.E., El-Mohri ,Y., Huang, W., Sandler, H.M., Yorkston, J., Scarpine, V.E., "Real-time, Digital Radiography and Fluoroscopy of human subjects using active-matrix, flat-panel imagers at diagnostic energies." 1996 Meeting of Radiological Society of North America, Chicago, IL, December 1-6, 1996, supplement to Radiology 201(P), pp. 327 (abstract). 